\title{
TRATAMENTO DE UM EFLUENTE MODELO TÊXTIL VIA REAÇÃO DE FENTON
}

Janara Aline Rocha ${ }^{1}$, Luciano André Deitos Koslowski ${ }^{2,3,4}$, Silvana Licodiedoff ${ }^{3}$, Humberto Gracher Riella ${ }^{3,4}$

${ }^{1}$ Universidade da Região de Joinville/UNIVILLE

${ }^{2}$ Universidade do Estado de Santa Catarina/UDESC

${ }^{3}$ Universidade Regional de Blumenau/FURB

${ }^{3}$ Programa de Pós Graduação em Engenharia Química e Alimentos/POSENQ

${ }^{4}$ Universidade Federal de Santa Catarina/UFSC

janara.alinne@gmail.com ${ }^{1}$

Artigo submetido em julho/2014 - Aceito em maio/2016

DOI: $10.15628 /$ holos.2016.2250

\section{RESUMO}

Nos últimos anos, a problemática de contaminações ambientais atingiu grandes proporções e as indústrias têxteis tem grande colaboração neste cenário, pois possuem um dos processos de maior geração de poluentes, contribuindo com uma carga poluidora rejeitada no meio ambiente, que quando não tratado corretamente são indutores de sérios problemas de contaminação ambiental. Os padrões de qualidade tornam-se gradativamente mais exigentes. Uma forma de tratar estes resíduos é pela técnica dos Processos Oxidativos Avançados (POA's), que são processos com potencial de produzir radicais hidroxila $(\bullet \mathrm{OH})$, espécies altamente oxidantes, capazes de melhorar a matéria orgânica. O presente estudo teve como objetivo avaliar a aplicação de um Processo Oxidativo Avançado no tratamento de efluentes têxteis, denominado processo oxidativo de Fenton. Nos estudos realizados, envolvendo a degradação dos corantes Azul turquesa e Verde malaquita, por meio de um planejamento fatorial, análises de absorbância, DQO e pH, ficou evidenciada a elevada capacidade de degradação da reação de Fenton. Os resultados obtidos demonstraram que em $\mathrm{pH}$ 3,0 e temperatura de $25^{\circ} \mathrm{C}$ a remoção de cor foi de $99,3 \%$ e a DQO de $85,7 \%$. A $50^{\circ} \mathrm{C}$ com o mesmo valor de $\mathrm{pH}$ a remoção de cor foi de $94,6 \%$, e a DQO de $92,8 \%$.

PALAVRAS-CHAVE: processos oxidativos avançados, POA's, efluente têxtil, reação de fenton.

\section{THE TREATMENT OF A MODEL TEXTILE EFFLUENT BY FENTON'S REACTION}

\begin{abstract}
The issue of environmental contamination has recently reached enormous proportions and textile industries have had a hand in this serious problem. They actually process more and more pollutants and thus contribute towards the disposal of a heavy pollution load into the environment. If not treated correctly, grave problems in environmental contamination ensue. Quality standards are becoming stricter. The technique of Advanced Oxidative Processes (AOPs) is one of the several ways to treat residues. The processes may produce hydroxyl radicals $(\bullet \mathrm{OH})$, highly oxidation species, capable of
\end{abstract}

improving organic matter. Current analysis evaluates the application of AOP in the treatment of textile effluents, called Fenton's oxidative process. The high capacity of degradation of Fenton's reaction has been evidenced in studies with the degradation of dyes turquoise blue and malachite green by a factorial plan, absorbance analysis, $\mathrm{COD}$ and $\mathrm{pH}$. Results show that color removal in $\mathrm{pH} 3.0$ at $25^{\circ} \mathrm{C}$ amounted to $99.3 \%$ and COD at $85.7 \%$. Color removal reached $94.6 \%$ and COD at $92.8 \%$ at $50^{\circ} \mathrm{C}$ and similar $\mathrm{pH}$ rate.

KEY-WORDS: advanced oxidative processes; AOP; textile effluents; Fenton's reaction. 


\section{INTRODUÇÃO}

O tratamento de efluentes na presença de corantes é uma preocupação crescente nos últimos anos na indústria têxtil devido à problemática do descarte dos banhos de tingimento residuais em um corpo hídrico receptor, bem como, de poluentes provenientes destes banhos de tingimento que podem ser apontados como substâncias potencialmente tóxicas conforme Kao (2001). O esgoto da indústria têxtil tem sido taxado como o mais poluente entre os setores industriais em termos de volume e composição de efluentes. Em adição ao efeito visual e ao efeito adverso dos corantes em termos de impacto de Demanda Química de Oxigênio (DQO), muitos corantes são tóxicos, mutagênicos e carcinogênicos (Peixoto, 2013).

$\mathrm{O} \mathrm{pH}$ do banho de tingimento pode afetar o comportamento do corante de várias maneiras. Assim, um corante pode ser convertido numa forma mais solúvel por ionização do grupo hidroxila, por exemplo, e mostrar um comportamento diferente durante o tingimento (Cogorni et al., 2014). Em condições extremas, certos corantes podem mesmo hidrolisar, mudando o seu comportamento e até a sua tonalidade ${ }^{3}$. Estudos indicam que o tingimento em tons médios e escuros nos tecidos de poliéster (PES), poliéster com elastano (PES/PUE) e poliamida (PA) resultam na diminuição do $\mathrm{pH}$ da água de tingimento de 4,0 para 3,5 conforme a proporção de água de reuso reportado por Cogorni et al. (2014).

Conforme Padoley et al. (2011), vários métodos de processos de oxidação avançados (POA's), como: fotodissociação direta, sistema ozônio/ultravioleta, fotocatálise com dióxido de titânio $\left(\mathrm{TiO}_{2}\right)$, sistema $\mathrm{H}_{2} \mathrm{O}_{2} / \mathrm{UV}$ e reagente de Fenton estão sendo estudados e aplicados para produzir intermediários altamente reativos capazes de oxidar a carga poluente, dentre estes o processo de Fenton demonstra-se vantajoso perante os demais por ter operação mais simples, custo de capital e operacional menores, além de não necessitar de irradiação, desta forma sem gasto de energia (Borba, 2011). Os POA's são caracterizados pela baixa seletividade do ataque dos radicais hidroxila, o que é uma característica muito importante para um oxidante utilizado no tratamento de efluentes (Jerônimo et al., 2014).

O reagente de Fenton isoladamente ou em combinação provou ser uma maneira eficiente para degradar poluentes orgânicos e tem sido utilizada para o tratamento de uma ampla variedade de efluentes industriais (Hermosilla et al., 2012). Contudo, para que a reação ocorra de forma mais eficaz, observou-se através de estudos que há fatores condicionantes da eficiência do processo (Selcuk et al., 2005): dosagem de peróxido de hidrogênio $\left(\mathrm{H}_{2} \mathrm{O}_{2}\right)$, concentração de ferro $\left(\mathrm{Fe}^{2+}\right), \mathrm{pH}$ e temperatura. Conforme Yingxun et al. (2006) a dosagem de peróxido de hidrogênio é determinante na degradação de corantes via reação de Fenton, já que vários estudos reportam a existência de uma condição ótima de $\mathrm{H}_{2} \mathrm{O}_{2}$, a uma razão molar $\mathrm{Fe}^{2+} / \mathrm{H}_{2} \mathrm{O}_{2}$, não havendo um consenso sobre a condição que fornece os melhores resultados (Chang et al., 2008).

O presente estudo tem como proposta analisar a partir de um planejamento fatorial a degradação de uma blenda de corantes (efluente modelo) Azul turquesa e Verde malaquita, pelo fato de não serem disponíveis na literatura estudos da reação de Fenton na degradação destes corantes utilizando diferentes relações de mistura. 


\section{MATERIAIS E MÉTODOS}

\subsection{PLANEJAMENTO FATORIAL ESTATÍSTICO}

De acordo com os fatores que interferem na reação de Fenton, foi elaborado um planejamento fatorial $2^{4}$ para testar e associar as variáveis (temperatura, $\mathrm{pH}$, concentração de peróxido de hidrogênio e concentração de sulfato ferroso) na degradação dos corantes Azul turquesa e Verde malaquita. A Tabela 1 apresenta as variáveis de estudo do processo, sendo o efeito negativo a relação menos favorável a degradação do corante e o efeito positivo como a relação mais favorável. A relação ótima de sulfato ferroso e peróxido de hidrogênio de 1:5 conforme mencionado na literatura (Gulkaya et al, 2006). A Tabela 2 apresenta o delineamento experimental em duplicata do estudo.

Tabela 1: Matriz do delineamento experimental utilizado.

\begin{tabular}{|c|c|c|c|}
\hline PARÂMETRO & UNIDADE & EFEITO NEGATIVO & EFEITO POSITIVO \\
\hline TEMPERATURA & ${ }^{\circ} \mathrm{C}$ & 25 & 50 \\
\hline $\mathrm{pH}$ & ------- & 4,0 & 3,0 \\
\hline $\begin{array}{c}\text { PERÓXIDO DE } \\
\text { HIDROGÊNIO }\end{array}$ & $\mathrm{mL}$ & 0,08 & 0,13 \\
\hline SULFATO FERROSO & $\mathrm{g} / \mathrm{L}$ & 0,053 & 0,089 \\
\hline
\end{tabular}

Tabela 2: Planejamento fatorial para a reação de Fenton.

\begin{tabular}{|c|c|c|c|c|}
\hline EXPERIMENTO & TEMPERATURA & $\mathbf{p H}$ & $\mathbf{H}_{\mathbf{2}} \mathbf{O}_{\mathbf{2}} \mathbf{( \mathbf { m L } )}$ & $\mathrm{FeSO}_{\mathbf{4}} \mathbf{( g / L} \mathbf{~}$ \\
\hline 1 & 25 & 3,0 & 0,13 & 0,089 \\
\hline 2 & 25 & 3,0 & 0,13 & 0,053 \\
\hline 3 & 25 & 4,0 & 0,13 & 0,089 \\
\hline 4 & 25 & 4,0 & 0,13 & 0,053 \\
\hline 5 & 25 & 3,0 & 0,08 & 0,053 \\
\hline 6 & 25 & 4,0 & 0,08 & 0,053 \\
\hline 7 & 25 & 4,0 & 0,08 & 0,089 \\
\hline 8 & 25 & 3,0 & 0,08 & 0,089 \\
\hline 9 & 50 & 3,0 & 0,13 & 0,089 \\
\hline 10 & 50 & 3,0 & 0,13 & 0,053 \\
\hline 11 & 50 & 4,0 & 0,13 & 0,089 \\
\hline 12 & 50 & 4,0 & 0,13 & 0,053 \\
\hline 13 & 50 & 3,0 & 0,08 & 0,053 \\
\hline 14 & 50 & 4,0 & 0,08 & 0,053 \\
\hline 15 & 50 & 4,0 & 0,08 & 0,089 \\
\hline 16 & 50 & 3,0 & 0,08 & 0,089 \\
\hline
\end{tabular}




\subsection{FORMULAÇÃO DO CORANTE E DETERMINAÇÃO DO COMPRIMENTO DE ONDA}

A solução de corante utilizada neste trabalho foi composta por dois corantes distintos, o verde malaquita e o azul turquesa, na concentração de $0,2 \mathrm{~g} / \mathrm{L}$, separadamente, e na forma de blenda 50/50 para realização dos experimentos.

Após a formulação do efluente modelo, este foi utilizado como padrão de referência para realizar a análise de espectrofotometria de varredura no campo de luz invisível, de forma a determinar o valor de absorbância a ser utilizado para análise das amostras.

\subsection{DELINEAMENTO DO EXPERIMENTO}

A reação de Fenton ocorre favoravelmente em meio ácido (Torrades, 2011), assim procedeu-se o ajuste do $\mathrm{pH}$ do efluente modelo com solução de ácido sulfúrico $1 \mathrm{M}$ de acordo com os valores estabelecidos na Tabela 1. Na etapa seguinte, adicionou-se sob agitação constante sulfato ferroso $\left(\mathrm{FeSO}_{4}\right)$ conforme apresentado na Figura 1. O $\mathrm{FeSO}_{4}$ é utilizado como catalisador desta reação com a finalidade de formar radicais hidroxila $(\bullet \mathrm{OH})$ por ser um oxidante forte $(E=2,73 \mathrm{~V})$, não seletivo e capaz de oxidar rapidamente uma ampla gama de compostos orgânicos poluentes ${ }^{12}$.

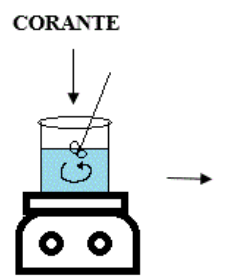

(A)

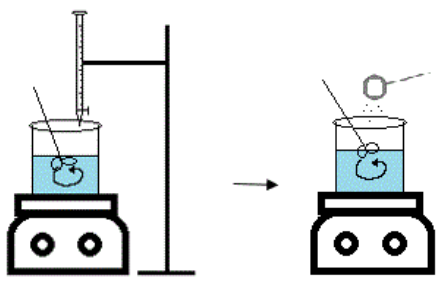

(B)
(C)

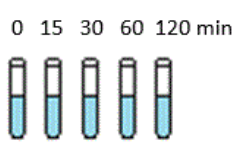

(F)
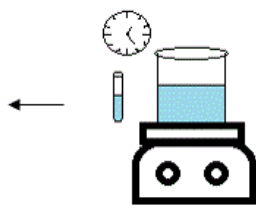

(E)

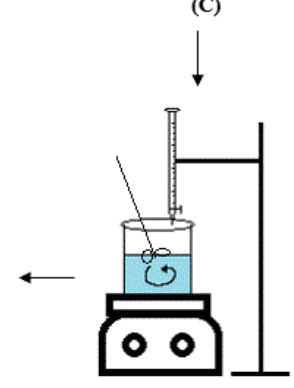

(D)

Figura 1: Ilustração sobre o procedimento experimental utilizado para a reação de Fenton.

Após o ajuste do $\mathrm{pH}$ e adição do $\mathrm{FeSO}_{4}$, adicionou-se o peróxido de hidrogênio nas concentrações estabelecidas na Tabela 1(Figura 1, D). Depois da adição do catalisador e do oxidante, a reação foi conduzida no intervalo de tempo de $120 \mathrm{~min}$, conforme modelo proposto por Martins (2011). Foram retiradas amostras nos intervalos de tempos de $0,15,30$, 60 e 120 min, para avaliação da reação de Fenton (Figura 1, E/F). 


\subsection{ANÁLISES DAS AMOSTRAS}

Após a retirada da amostra, procedeu-se o ajuste do $\mathrm{pH}$ do efluente para 7,0 com solução de hidróxido de sódio $0,1 \mathrm{M}$, de forma que a reação fosse interrompida no tempo determinado. Realizado o ajuste do $\mathrm{pH}$, as amostras foram mantidas em repouso por 4 horas (Ponezi, 2000), e posteriormente, foram analisadas quanto a valores de absorbância e de DQO.

Para a quantificação da eficiência dos tratamentos pesquisados, quando se trata de redução de cor, costuma se utilizar a análise do espectro de absorção de luz visível da amostra pesquisada (Marmagne \& Coste, 1996). A medição foi feita em espectrofotômetro Shimadzu UV-160A com comprimento de onda de $335 \mathrm{~nm}$. O ajuste inicial foi realizado empregando como "branco", água, nos dois compartimentos e regulando-se o aparelho para absorbância zero. As leituras foram feitas substituindo-se o "branco" do compartimento pelas amostras a serem medidas.

\subsection{DEMANDA QUÍMICA DE OXIGÊNIO (DQO)}

A DQO é a quantidade de oxigênio necessária para oxidação de matéria orgânica através de um agente químico. O aumento na concentração de DQO num corpo d'água se deve principalmente a despejos de origem industrial, sendo um parâmetro indispensável na caracterização de esgotos sanitários e efluentes industriais (CETESB, 2009). No caso de amostras provenientes da degradação, a análise da DQO se torna relevante, pois indica a eficiência do tratamento e em qual nível de mineralização a amostra se apresenta (Stylid et al., 2003).

A determinação da DQO foi realizada para as amostras que apresentaram um índice de redução de cor superior a 95\%. Este teste foi realizado empregando o kit para DQO Hach 0$1500 \mathrm{mg} / \mathrm{L}$, para leitura colorimétrica no espectrofotômetro DR/ $4000 \mathrm{U}$ da marca Hach. A análise foi realizada com a adição de $2 \mathrm{~mL}$ da amostra no tubo de ensaio contendo o reagente DQO 0-1.500 mg/L, com agitação da mistura e posterior digestão da amostra no reator Hach DRB 200 a $165^{\circ} \mathrm{C}$ no intervalo de tempo de 2 horas. Na etapa seguinte, retirou-se o tubo de ensaio do aparelho para resfriar e fez-se a leitura da DQO.

\section{RESULTADOS E DISCUSSÕES}

\subsection{CARACTERIZAÇÃO DO EFLUENTE MODELO}

Os corantes utilizados nos experimentos foram verde malaquita $\left(\mathrm{C}_{23} \mathrm{H}_{26} \mathrm{~N}_{2} \mathrm{O}\right)$ também chamado verde básico 4-1 trifenilmetano amplamente utilizado na indústria, principalmente no processo de tingimento de materiais como seda, couro e papel, sendo atualmente, alvo de preocupações ambientais, pois em soluções aquosas, possuem propriedades que tornam difícil a remoção (Ding, 2012); e o corante azul turquesa $\left(\mathrm{C}_{32} \mathrm{H}_{16} \mathrm{CuN}_{8} \mathrm{Na}_{2} \mathrm{O}_{6} \mathrm{~S}_{2}\right)$, da família dos corantes azóicos que são considerados os mais tóxicos para a biota aquática e, há descrições de efeitos carcinogênicos em humanos (Ninupriya, 2008). As quebras das ligações destes 
corantes formam produtos carcinogênicos, sendo oportuna a remoção destes corantes para sua disposição nos corpos receptores.

As Figuras 2, 3 e 4 apresentam a varredura feita para a determinação do comprimento de onda a ser utilizado no experimento. A Figura 2 apresenta a curva de varredura para o corante verde malaquita com pico em $620 \mathrm{~nm}$ e $0,251 \mathrm{~A}$ como valor máximo de absorbância. $A$ Figura 3 apresenta a curva de varredura para o corante azul turquesa com obtenção do pico em $335 \mathrm{~nm}$ e 0,513A. A Figura 4 apresenta a curva de varredura para a blenda de corantes (Verde malaquita e Azul turquesa), com concentração de $0,2 \mathrm{~g} / \mathrm{L}$, observando-se que dois picos de absorbância máxima (335 nm - 0,519A e 622 nm - 0,389A).

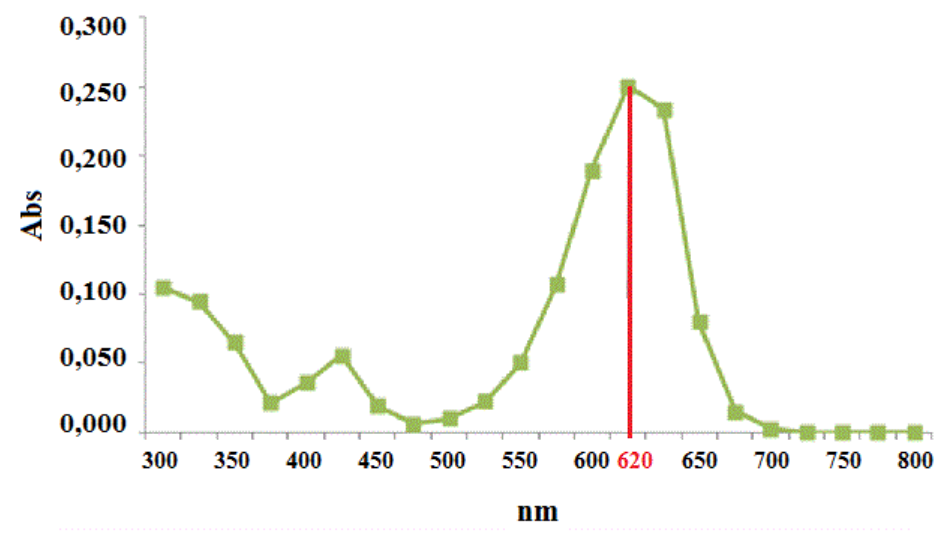

Figura 2: Curva de varredura para o corante Verde Malaquita.

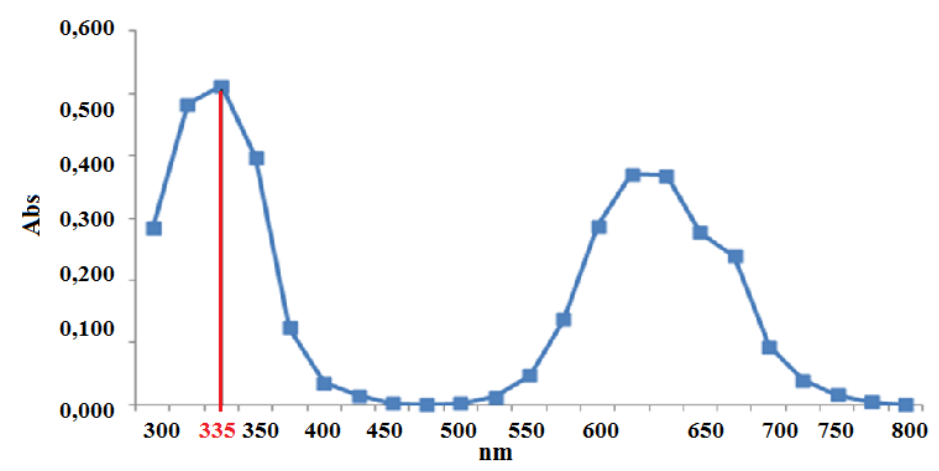

Figura 3: Curva de varredura para o corante Azul Turquesa.

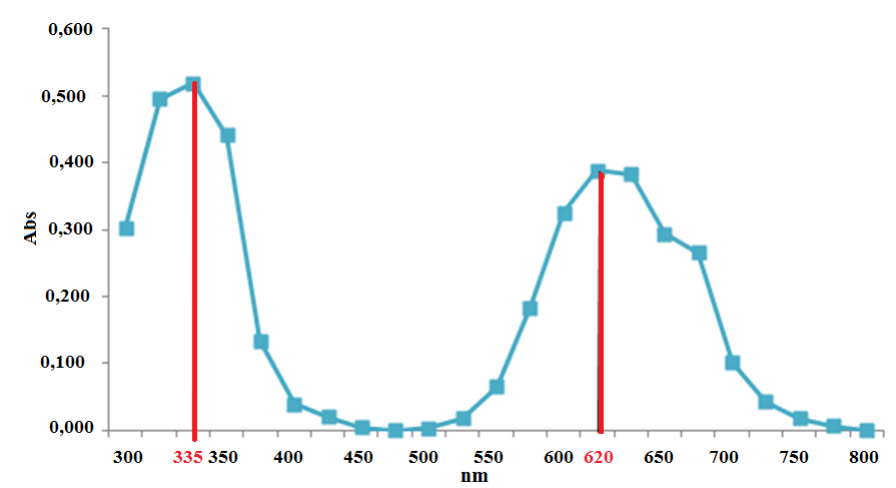

Figura 4: Curva de varredura para a blenda Verde Malaquita/Azul Turquesa. 


\subsection{OTIMIZAÇÃO DA RELAÇÃO $\mathrm{Fe}^{2+} / \mathrm{H}_{2} \mathrm{O}_{2}$}

A relação $\mathrm{Fe}^{2+} / \mathrm{H}_{2} \mathrm{O}_{2}$ é muito importante para a otimização da reação, sendo observado que o objetivo consiste mensurar a concentração de catalisador necessária a ser adicionada para a máxima produção de radicais hidroxil $(\bullet \mathrm{OH})$.

O processo Fenton pode apresentar diferentes funções de tratamento, dependendo da relação entre o peróxido de hidrogênio e sulfato de ferro (Neyens, 2003). Quando a relação de $\mathrm{H}_{2} \mathrm{O}_{2}$ excede a de $\mathrm{Fe}^{2+}$, o tratamento tende a ter efeito oxidante (Idil, 2007). No sentido inverso, o tratamento assume caráter de coagulação química. Assim como a oxidação, a função coagulante é inerente ao reagente de Fenton, sendo principalmente devida à formação de complexos hidróxidos-férricos (Neyens, 2003).

Conforme estudos realizado por Koprivanac et al. (2006), a relação ótima entre $\mathrm{Fe}: \mathrm{H}_{2} \mathrm{O}_{2}$ é de 1:5, então empregou-se esta relação para a modelagem experimental. Porém, pode-se observar que esta relação tem um limite mínimo para a atuação oxidante em relação à massa dos reagentes. Os modelos experimentais 1 e 5 justificam a relação $\mathrm{Fe}: \mathrm{H}_{2} \mathrm{O}_{2}$ de $1: 5$ pelos valores médios de absorbância que foram obtidos a temperatura de $25^{\circ} \mathrm{C}$ e pH 3,0.

A Tabela 3, a seguir, apresenta os valores médios da absorbância em função do tempo e temperatura de reação com a respectiva remoção de cor (\%) conforme o delineamento experimental definido.

Tabela 3: Valores de absorbância (335 nm) para planejamento experimental.

\begin{tabular}{|c|c|c|c|c|c|c|}
\hline EXPERIMENTO & $\begin{array}{c}\text { tempo } \\
0 \mathrm{~min}\end{array}$ & $\begin{array}{l}\text { tempo } \\
15 \mathrm{~min}\end{array}$ & $\begin{array}{l}\text { tempo } \\
30 \mathrm{~min}\end{array}$ & $\begin{array}{l}\text { tempo } \\
60 \mathrm{~min}\end{array}$ & $\begin{array}{c}\text { tempo } \\
120 \mathrm{~min}\end{array}$ & $\begin{array}{c}\text { Redução } \\
\text { Cor (\%) } \\
(120 \text { min })\end{array}$ \\
\hline 1 & 2,483 & 0,267 & 0,237 & 0,037 & 0,017 & 99,3 \\
\hline 2 & 2,483 & 1,047 & 0,948 & 0,826 & 0,476 & 80,8 \\
\hline 3 & 2,483 & 0,239 & 0,177 & 0,071 & 0,021 & 99,2 \\
\hline 4 & 2,483 & 1,227 & 1,181 & 0,550 & 0,357 & 85,6 \\
\hline 5 & 2,483 & 1,469 & 1,269 & 0,855 & 0,805 & 67,6 \\
\hline 6 & 2,483 & 1,530 & 1,164 & 1,037 & 0,968 & 61,0 \\
\hline 7 & 2,483 & 1,907 & 1,617 & 1,242 & 1,030 & 58,5 \\
\hline 8 & 2,483 & 1,724 & 1,336 & 1,159 & 0,918 & 63,1 \\
\hline 9 & 2,483 & 0,347 & 0,231 & 0,174 & 0,133 & 94,6 \\
\hline 10 & 2,483 & 0,542 & 0,454 & 0,312 & 0,217 & 91,3 \\
\hline 11 & 2,483 & 0,281 & 0,156 & 0,125 & 0,074 & 97,0 \\
\hline 12 & 2,483 & 1,198 & 0,836 & 0,572 & 0,306 & 87,7 \\
\hline 13 & 2,483 & 1,943 & 1,668 & 1,501 & 1,478 & 40,5 \\
\hline 14 & 2,483 & 1,661 & 1,558 & 1,527 & 1,175 & 52,7 \\
\hline 15 & 2,483 & 1,625 & 1,017 & 0,799 & 0,659 & 73,5 \\
\hline 16 & 2,483 & 1,275 & 0,894 & 0,859 & 0,777 & 68,7 \\
\hline
\end{tabular}


De acordo com o experimento 1, empregando a relação de $0,13 \mathrm{~mL}$ de peróxido de hidrogênio e 0,089 g de sulfato de ferro, observou-se um valor de absorbância de 0,267 nm em 15 minutos de reação, enquanto para o experimento 5 , com $0,08 \mathrm{~mL}$ de peróxido de hidrogênio e $0,053 \mathrm{~g}$ de sulfato de ferro, apresentou valor de 1,469 $\mathrm{nm}$ para o mesmo período de tempo de reação. Sendo assim, percebe-se que há uma massa mínima de reagentes para promover a oxidação dos compostos.

O índice de redução de cor após 120 minutos no experimento 5, que possui relação 1:5 apresenta menor percentual de redução de cor (67,6\%) quando comparado com os experimentos 1 e 3 (99\%), denotando uma concordância no que diz respeito a relação ideal de sulfato ferroso e peróxido de hidrogênio.

Desta forma, pode-se observar que para uma maior eficiência na redução de cor, devese optar por utilizar maior temperatura $\left(50^{\circ} \mathrm{C}\right)$ e $\mathrm{pH}(4,0)$ para promover uma redução da cor, e também maior concentração de $\mathrm{FeSO}_{4}$ e $\mathrm{H}_{2} \mathrm{O}_{2}$ para maximizar a remoção de cor ao longos dos 120 min de reação de Fenton.

A partir dos dados de redução de cor obtidos, optou-se por fazer análise de DQO apenas com os experimentos onde a redução de cor foi igual ou superior a 95\%. Neste caso, foram analisados os experimentos 1, 39 e 11. Os experimentos 1, 3, 9 e 11 apresentam uma relação de $\mathrm{Fe}^{2+} / \mathrm{H}_{2} \mathrm{O}_{2}$ de 1:5 com valores de $0,13 \mathrm{ml}$ de peróxido de hidrogênio e $0,089 \mathrm{~g}$ de sulfato de ferro, conduzindo a uma redução de 85 a 95\% da remoção de cor após 120 minutos de reação como pode ser observado na Tabela 4.

Tabela 4: Redução da DQO em 120min de reação.

\begin{tabular}{|c|c|c|c|c|c|c|}
\hline EXPERIMENTO & $\begin{array}{c}\text { tempo } \\
0 \mathrm{~min}\end{array}$ & $\begin{array}{l}\text { tempo } \\
15 \mathrm{~min}\end{array}$ & $\begin{array}{l}\text { tempo } \\
30 \mathrm{~min}\end{array}$ & $\begin{array}{l}\text { tempo } \\
60 \mathrm{~min}\end{array}$ & $\begin{array}{l}\text { tempo } \\
120 \mathrm{~min}\end{array}$ & $\begin{array}{c}\text { Redução } \\
\text { Cor (\%) } \\
(120 \text { min) }\end{array}$ \\
\hline 1 & 269 & 209 & 178 & 91 & 39 & 85,7 \\
\hline 3 & 269 & 101 & 82 & 49 & 34 & 87,5 \\
\hline 9 & 269 & 190 & 151 & 102 & 20 & 92,8 \\
\hline 11 & 269 & 92 & 26 & 17 & 13 & 95,4 \\
\hline
\end{tabular}

Comparando os resultados dos experimentos 1, 3, 9 e 11 (Tabela 4), observa-se uma maior influência da ação da temperatura para redução de valores de DQO, comparada a mudança de $\mathrm{pH}$, sendo que a temperatura de $50^{\circ} \mathrm{C}$ permitiu uma maior redução da DQO, tanto para pH 3,0 (Experimento 9 - 93\%) quanto para a reação em pH 4,0 (Experimento 11 95\%).

Os experimentos que apresentaram valores mais significativos de redução de cor (99\%) foram os ensaios 1 e 3, conduzidos a $25^{\circ} \mathrm{C}$ com pH de 3,0 e 4,0, respectivamente. Para a redução de DQO, os experimentos com maiores valores de redução foram os ensaios 9 e 11 (93\% e 95\%, respectivamente), conduzidos em $\mathrm{pH} 3,0$ e 4,0, respectivamente, com 
temperatura de $50^{\circ} \mathrm{C}$. A relação entre as reduções de cor e DQO em função das variáveis temperatura e $\mathrm{pH}$ podem ser observadas na Figura 5.

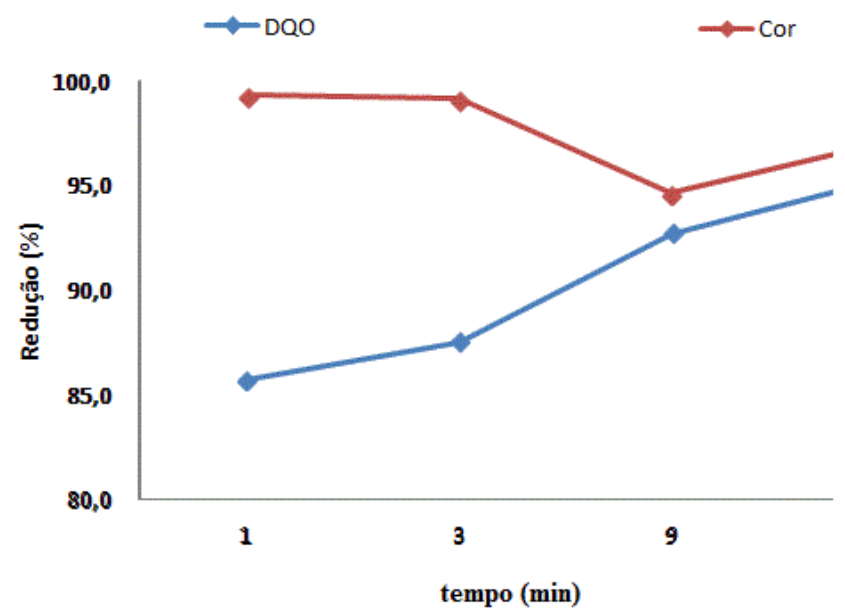

Figura 5: Redução de cor e de DQO em função de temperatura e pH.

Conforme apresentado na Figura 5, percebe-se a que o $\mathrm{pH}$ foi um parâmetro pouco significativo nos ensaios realizados, sugerindo-se então o uso do $\mathrm{pH} 4,0$, que apresentou valores de $95 \%$ de redução de $\mathrm{DQO}$ e $97 \%$ de redução de cor a $50^{\circ} \mathrm{C}$ e $87 \%$ e $99 \%$, respectivamente, a $25^{\circ} \mathrm{C}$. Uma justificativa para a situação descrita na Figura 5 , seria o fato que mesmo obtendo-se uma redução da cor, ainda permanece uma quantidade residual de compostos orgânicos em suspensão na amostra.

Em relação à temperatura, para os experimentos conduzidos em $\mathrm{pH} 4,0$, o maior valor de redução de cor foi obtido a $25^{\circ} \mathrm{C}$ e o maior valor de redução de DQO foi a $50^{\circ} \mathrm{C}$ (Figura 5). Como o efluente industrial a ser tratado normalmente se encontra em temperaturas superiores a $40^{\circ} \mathrm{C}$, e a $50^{\circ} \mathrm{C}$ (Experimento 11 - Figura 7) apresentou $97 \%$ de redução de cor, observa- que não há necessidade de resfriar o efluente para fazer o tratamento por reação de Fenton. Neste contexto, o valor de pH deve ser corrigido para 4,0, pois o efluente apresenta $\mathrm{pH}$ alcalino.

Desta forma, sugere-se para o tratamento de efluentes têxteis a partir do processo Fenton, uma relação 1:5 de $\mathrm{Fe}: \mathrm{H}_{2} \mathrm{O}_{2}$, ou seja $0,089 \mathrm{~g}$ e $0,13 \mathrm{~mL}$ em $\mathrm{pH} 4,0$ e temperatura de $50^{\circ} \mathrm{C}$ para $300 \mathrm{~mL}$ de efluente modelo utilizado neste trabalho, como aquela que fornece a melhor eficiência em remoção de cor e DQO.

\section{CONSIDERAÇÕES FINAIS}

Nos estudos realizados, envolvendo a degradação de uma blenda (mistura) de corantes de (Verde malaquita e Azul turquesa) ficou demonstrada a elevada capacidade de degradação do processo de Fenton. Observou-se que a partir da relação empregada de 1:5 $\mathrm{Fe}: \mathrm{H}_{2} \mathrm{O}_{2}$, tem um limite mínimo para a atuação oxidante em relação a massa de $\mathrm{FeSO}_{4}$ e $\mathrm{H}_{2} \mathrm{O}_{2}$, sendo estes de 0,089 g de sulfato de ferro e 0,13 $\mathrm{mL}$ de peróxido de hidrogênio, onde obtevese, aproximadamente, $90,4 \%$ de redução de cor em apenas 15 minutos. 
No tempo de 120 minutos, o experimento conduzido em pH 4,0 a 25으 obteve um valor de redução de cor de $99 \%$ e a $50 \circ \mathrm{C}$ este valor foi de $97 \%$. Para os experimentos que apresentaram redução de cor acima de $95 \%$ em 120 minutos foram feitas as análises de DQO observando-se um valor de redução de DQO de $95 \%$ para experimentos conduzidos em pH 4,0 e $50^{\circ} \mathrm{C}$. Desta forma, sugere-se para tratamento de efluentes têxteis com o processo de Fenton, uma relação 1:5 de Fe: $\mathrm{H}_{2} \mathrm{O}_{2}$ em pH 4,0 e temperatura de $50^{\circ} \mathrm{C}$.

\section{REFERÊNCIAS BIBLIOGRÁFICAS}

1. BINUPRIYA, A.R.; SATHISHKUMAR, M.; SWAMINATHAN, K.U.; YUN, S.E. Comparative studies on removal of Congo red by native and modified mycelial pellets of Trametes versicolor in various reactor modes. Biresour Technol. v. 99. p. 1078-1080, 2008.

2. BORBA, F. H. Aplicação dos processos foto-Fenton e eletrofloculação no tratamento de efluente de curtume. Dissertação de Mestrado - Faculdade de Engenharia Química - Universidade Estadual do Oeste do Paraná, 2010.

3. CETESB. Companhia de Tecnologia de Saneamento Ambiental. Significado Ambiental e Sanitário das variáveis de qualidade de águas e dos sedimentos e metodologias analíticas e de amostragem, 2009.

4. CHANG, C. Effect of $\mathrm{pH}$ on Fenton process using estimation of hydroxyl radical with salicylic acid as trapping reagent. Water Science e Technology - WST. p. 873-879, 2008.

5. COGORNI, A.; CORREIA, C.H.G.; FRANÇA, P.H.; LICODIEDOFF, S.; KOSLOWSKI, L.A.D. Water reuse in the textile industry's dyeing process. 40 Congresso Internacional de Tecnologias para o Meio Ambiente, Anais do Congresso Internacional de Tecnologia para o meio Ambiente, INSS 21772916. v. 1. p. 1-7, abr. 2014.

6. DING, F. et al. Potential toxicity and affinity of triphenylmethane dye malachite green to lysozyme. Ecotoxicology and Environmental Safety, New York. v. 78, p. 41-49, 2012.

7. GULKAYA, I; SURUCU, G. A.; DILEK, F. B. Importance of $\mathrm{H}_{2} \mathrm{O}_{2} / \mathrm{Fe}^{2+}$ ratio in Fenton's treatment of a carpet dyeing wastewater. Journal of Harzadous Materials. v. 136. p. 763-769, 2006KAO, C. M.; CHOU, M.; FANG, W. L.; HUANG, B. Regulating colored textile wastewaters by $3 / 31$ wavelength ADMI methodsin Taiwan. Chemosphere. Vol. 44. p. 1055-1063, 2001.

8. HERMOSILLA, D.; MERAYO, N.; ORDÓÑEZ, R.; BLANCO, A. Optimization of conventional Fenton and ultraviolet-assisted oxidation processes for the treatment of reverse osmosis retentate from a paper mill. Waste Management. Vol. 32, p. 1236-1243, 2012.

9. IDIL A.; TEKSOY S. Acid dyebath effluent pretretment using Fenton's reagent: Process optimization, reaction kinetics and effect on acute toxicity. Dyes and Pigments. v. 73, p. 31-39, 2007.

10. JERÔNIMO, C.E.M.; FERNANDES, H.G.; MELO, H.S.N.; SOUSA, J.F. Estudo Comparativo de Técnicas Fotoquímicas aplicados ao tratamento de efluentes do beneficiamento da castanha de caju. Revista Holos, Ano 30, v. 1. p. 96-104, 2014.

11. KAO, C. M.; CHOU, M.; FANG, W.L.; HUANG, B. Regulating colored textile wastewaters by $3 / 31$ wavelength ADMI methodsin Taiwan. Chemosfere. V.44. p. 1055-1063, 2001.

12. KOPRIVANAC, N.; HRYOJE K.; BOZIC A. L. Fenton type processes for minimization of organic content in coloured wastewaters: Part I: Processes optimization. Dyes and Pigments. v. 74. p. 380387, 2006. 
13. MARMAGNE, O.; COSTE, C. Color Removal from textile plant effluents. American Dyestuff Reporter. v. 85. p. 15-21, 1996.

14. MARTINS, L. Estudo da aplicação de processos oxidativos avançados no tratamento de efluentes têxteis visando seu reuso, Mestrado em Desenvolvimento e Meio Ambiente - Universidade Federal do Piauí, 2011.

15. NEYENS, E.; BAEYENS, J. A Review of Classic Fenton's Peroxidation as an Advanced Oxidation Technique. Journal of Hazardous Materials. p.33-50, 2003.

16. PADOLEY, K. V.; MUDLIAR, S. N.; BANERJEE, S. K.; DESHMUKH, S. C. PANDEY, R. A. Fenton oxidation: a pretreatment option for improved biological treatment of pyridine and 3cyanopyridine plant wastewater. Chemical Engineering Journal. v. 166. p. 1-9, 2011.

17. PEIXOTO, F., MARINHO, G., RODRIGUES, K. Corantes Têxteis: Uma Revisão. Revista Holos, Ano 29, v. 5. p. $98-106,2013$.

18. PONEZI, A. N. Tratamento de efluente líquido da indústria cítrica por lodo ativado por batelada. Departamento de Engenharia Civil, Arquitetura e Urbanismo da Universidade Estadual de Campinas, 2000.

19. SELCUK, H. Decolorization and detoxification of textile wastewater by ozination and coagulation processes. Dyes and Pigments, v.64, p.217-222, 2005.

20. STYLID, M.; KONDARIDES, I.; VERYKIOS, E. Pathways of solar light induced photocatalytic degradation of azo dyes in aqueous $\mathrm{TiO}_{2}$ suspension. Applied Catalysis B: Environmental v. 40, p. $271-286,2003$.

21. TORRADES, F.; SÁIZ, S. e GARCIA, J. Using central composite experimental design to optimize the degradation of black liquor by Fenton reagent. Desalination. v. 268, p. 97-102, 2011.

22. YINGXUN, D.; MINGHUA, Z.; LECHENG, L. Role of the intermediates in the degradation of phenolic compounds by Fenton-like process. Journal of Hazardous Materials., 859-865, 2006. 